EESTI NSV TEADUSTE AKADEEMIA TOIMETISED, 29, KOIDE FUOSIKA * MATEMAATIKA. 1980, NR. 1

НЗВЕСТИЯ АКАДЕМИИ НАУК ЭСТОНСКОИ ССР. ТОМ 29 ФИЗИКА * МАТЕМАТИКА, 1980, № 1

\title{
ПРИБЛИЖЕННЫЙ МЕТОД РАСЧЕТА ПОЛЕЙ ТЕМПЕРАТУР И ТЕРМИЧЕСКИХ НАПРЯЖЕНИИ В СТЕНКЕ ТРУБ ПРИ РЕЗКОМ ОХЛАЖДЕНИИ
}

\author{
(Представлена И. Эпиком)
}

В настоящее время большой технический интерес представляет решение проблемы расчета полей температур и термических напряжений в стенке труб при их резком охлаждении. Такие вопросы, например, имеют место при водяной обмывке труб поверхностей нагрева парогенераторов. Вследствие резкого охлаждения в поверхностном слое металла могут появиться значительные термические напряжения, могущие обусловить возникновение термоусталостных трещин.

Известно много теоретических решений задач по определению нестационарного температурного поля в цилиндре и стенке цилиндрической трубы при равномерном нагреве (охлаждении) их поверхностей $[1,2]$. Недостатками приведенных в этих работах формул являются сложность и невозможность выражения их через элементарные функции. Вследствие этого не могут быть получены в общем виде и формужы поля термических напряжений. Следовательно, использование этих формул в практике инженерных расчетов связано с большими трудностями. Қак показывает опыт, охлаждение нагретых поверхностей в случае обмывки их струями жидкости, происходит не равномерно, а локализованно, т: е. в отдельных точках поверхности. Поэтому тепловые потоки в стенке трубы имеют не только радиальные, но и окружное и осевое направления. Поэтому расчет температурных полей в стенке трубы в предположении равномерного охлаждения всей поверхности может дать в рассматриваемом случае определенные отклонения от действительности.

Ниже описывается приближенный метод расчета полей температур в стенке трубы при ее резком охлаждении, а затем, на фснове полученных полуэмпирических формул, выводятся упрощенные зависимости для определения термических напряжений.

\section{Опытная установка и методика исследований}

Экспериментальное исследование полей температур в стенке трубы при резком локальном охлаждении ее водой-проводилось в специальной полностью автоматизированной установке: Опытными элементами служили четыре образца длиной 110 мм, вырезанные из трубы диа- 
метром $32 \times 6$ мм (сталь $12 X 1 M \Phi)$. Спаи термопар отстояли от внешней поверхности трубы $(x=0)$ на расстояниях $x=0 ; 0,5 ; 1,5$ и 3,1 мм. Кабельные термопары КТМС градуировки ХК с термоэлектродами диаметром 0,2 мм крепились ко дну отверстий серебряным припоем.

Дистиллированная вода впрыскивалась через сопло постоянного сечения с помощью плунжерного насоса. Изменение удельной водяной нагрузки $m_{F}$ (массы воды, поступающей в единицу времени на единицу поверхности измерительного элемента) или динамического напора $H_{g}$ обеспечивалось в широких пределах путем регулирования напряжения в соленоиде насоса.

Начало впрыска воды регистрировалось по появлению тока в цепи электрода, установленного перед соплом. Этот момент, а также изменение температуры в стенке трубы фиксировались самопишущим многоканальным осциллографом Н-327. Точность измерения температур составляла $\pm 2,5^{\circ} \mathrm{C}$, времени $\pm 0,01$ сек.

Влияние отверстия под термопару учитывалось путем «снятия» теплового потока, соответствуюшего донышку отверстия

$$
Q_{\text {от } \mathrm{B}}=\lambda \frac{\pi d_{0}^{2}}{4}\left(\frac{\partial \Delta t(x, \tau)}{\partial x}\right)_{x=x_{1}}
$$

где $\left(\frac{\partial \Delta t(x, \tau)}{\partial x}\right)_{x=x_{1}}-$ градиент температуры в сечении донышка отверстия, $x_{1}$ - расстояние донышка отверстия термопары от внешней поверхности трубы, $\lambda$ - коэффициент теплопроводности металла, $d_{0}$ - диаметр отверстий, $\tau-$ время контакта.

В результате получено, что относительная погрешность показаний термопары, обусловленная сверлением, имеет вид

$$
\varphi=c d_{0}^{2}\left[8 \tau^{b} \sqrt{\pi a \tau_{*}}\right]^{-1} \operatorname{arctg} \sqrt{\tau / \tau_{*}},
$$

где $a-$ коэффициент температуропроводности; $\tau$ * - постоянная, имеющая размерность времени и зависящая от формы кривой распределения теплового потока на поверхности; $b, c$ - постоянные.

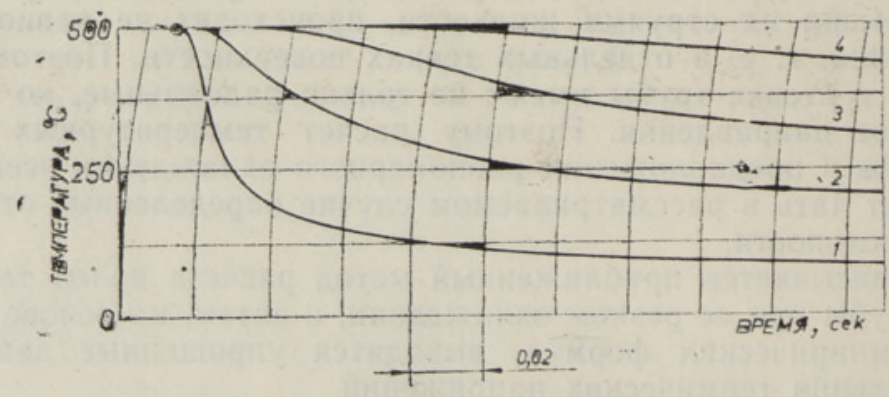

Рнс. 1. Характер изменения температуры внутри стенки образца (осциллограмма): $\bigcirc$ - начальный момент впрыска

$$
\text { воды; } x=0 \text { (1), } 0,5 \text { (2), } 1,5 \text { (3) и } 3,1 \text { м. (4). }
$$

Как видно из формулы (1), величина ф зависит только от диаметра отверстия и длительности контакта. Расчет показывает, что при $d_{0}=$ $=1,6$ мм величина $\varphi \approx 0,08$. 
В качестве примера на рис. 1 показано изменение температуры в стенке трубы при ее обмывке водой.

Разработанная экспериментальная методика позволила получить при заданных начальной температуре образца $\left(t_{0}=400,450,500\right.$ и $\left.550{ }^{\circ} \mathrm{C}\right)$, диаметре сопла $\left(d_{\mathrm{c}}=2,5\right.$ и $\left.4,0 \mathrm{MM}\right)$ и удельной водяной нагрузке $\left(m_{F}=400-1300 \kappa 2 /\left(M^{2} \cdot\right.\right.$ сек $\left.)\right)$ зависимость вида $\Delta t(x, \tau)=$ $=t_{0}-t(x, \tau)$.

\section{Температурное поле в стенке трубы при ее резком охлаждении}

В основу принят метод расчета тепловы: процессов в условиях лока.льного охлаждения (нагревания) поверхности в предположении, что длительность охлаждения очень мала (не превышает нескольких десягых долей секунды), так что изменение температурного поля локализуется в небольшом объеме металла вблизи места охлаждения, размеры котоporo значительно меньше наружного радиуса трубы. Это упрощает задачу и позволяет рассматривать поверхность охлаждаемой трубы как поверхность полубесконечного тела, не имеющего теплообмена с окружающей средой (кроме пятна охлаждения).

Предположим, что распределение теплового потока на поверхности $q(R)$ при ее точечном охлаждении выражается известным экспоненциальным законом

$$
q(R)=q_{\text {мakc }} \mathrm{e}^{-K R^{2}},
$$

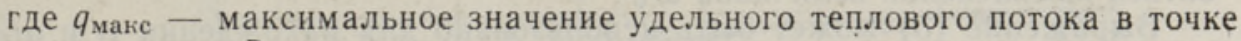
охлаждения; $R$ - расстояние от точечного источника охлаждения до данной точки на поверхности; $K$ - коэффициент, характеризующий форму кривой распределения теплового потока на поверхности. В этом случае температурное поле в теле описывается уравнением [3]

$$
d t(x, R, \tau)=\exp \left(-\frac{x^{2}+R^{2}}{4 a \tau}\right)\left[4 \pi \lambda \tau^{2 / 3} \sqrt{\pi \mathrm{a}}\right]^{-1} d Q
$$

где $Q$ - количество мгновенно подведенного к телу тепла (или отнятого от него); $x$ - расстояние от точки охлаждения на поверхности до рассматриваемой точки внутри стенки трубы.

Рассматривая тепловой поток от поверхности к струе как интегральную сумму тепловых потоков мгновенных точечных источников $d Q=$ $=q(R) d \tau$ и интегрируя результат с учетом (2) по времени $\tau$, получим уравнение, описывающее изменение температуры в теле на расстоянии $x$ по перпендикуляру от центра пятна охлаждения $(R=0)$ :

$$
\Delta t(x, \tau)=\alpha r_{0}^{2} \delta t(4 \lambda \sqrt{\pi a})^{-1} \int_{0}^{\tau} \exp \left(-\frac{x^{2}}{4 a \tau}\right)\left[\sqrt{\tau}\left(\tau+\tau_{*}\right)\right]^{-1} d \tau .
$$

В формуле, (3) учтено, что изменение температуры в теле обусловлено источником охлаждения тепловой мощностью

$$
Q=\alpha r_{0}^{2} \pi\left(t_{0}-t_{\text {ж}}\right)=\alpha r_{0}^{2} \pi \delta t .
$$

Здесь $\alpha-$ условный коэффициент теплоотдачи от поверхности к воде, $r_{0}$ - радиус пятна охлаждения на поверхности тела, $t_{0}$ - первоначаль-

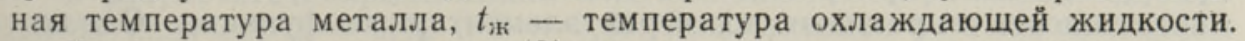

В общем виде уравнение (3) не интегрируется через элементарные функции, но легко решается для частного случая $x=0$, что позволяет 


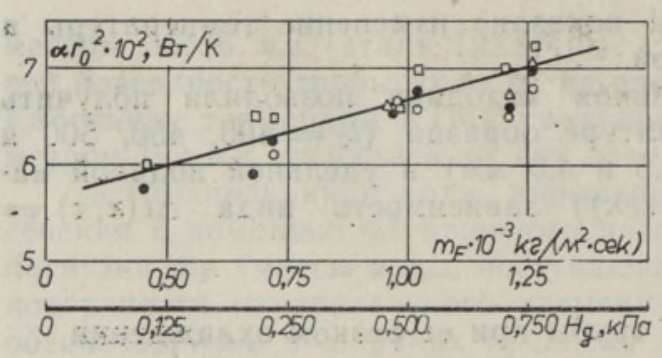

Рис. 2. Зависимость $\alpha r_{0}^{2}$ от $m_{F}$ или от $H_{\mathrm{g}}$ в пятне охлаждения: $t_{0}=400(\mathrm{O})$, $450(\triangle), 500(\square)$ и $550^{\circ} \mathrm{C}($ (3)

получить закономерность изменения температуры на внешней поверхности тела в центре пятна охлаждения:

$$
\Delta t(0, \tau)=\frac{\alpha r_{0} \delta t}{4 \lambda \sqrt{\pi a}} \int_{0}^{\tau} \frac{d \tau}{\left.\sqrt{\tau(\tau}+\tau_{*}\right)}=\frac{\alpha r_{0}^{2} \delta t}{2 \lambda \sqrt{\pi a \tau_{*}}} \operatorname{arctg} \sqrt{\frac{\tau}{\tau_{*}}} .
$$

Қак видно, велнчина $\Delta t(0 ; \tau)$ меняется во времени по закону $\operatorname{arctg} \sqrt{\tau / \tau}$. и н мере роста $\tau$-асимनтотически приблйжается к значению $\alpha r_{0}^{2} \tau \delta t\left(4 \lambda \overline{\sqrt{\pi a \tau_{*}}}\right)^{-1}$.

Следует отметить, что в рассматриваемом случае $\alpha$ является условной, меняющейся в пределах пятна охлаждения величиной. Возможнӧсть е точного определения путем непосредственных замеров предстайяется маловероятной. Трудно поддающимся непосредственному определению параметром является также $r_{0}$. Поэтому для характеристики интенсивности охлаждения в пятне более целесообразно пользоваться комплексной величиной $\alpha r_{0}^{2}$.

. Выражение (5) может быть использовано для нахождения $\tau$. и $\alpha r_{0}^{2}$ по опытным данным. Расчеты на ЭВМ показали, что для стали $12 \mathrm{X} 1 \mathrm{M} \Phi \tau_{*}=0,05$ сек.

: Комплексная величина $\alpha r_{0}^{2}$ несколько растет с увеличением-удельной водяной нагрузки $m_{F}$ или динамнческого напора струи $H_{g}$ в пятне охлаждения и от начальной температуры металла практически не зависит (см. рис. 2).

При выводе формулы (3) предполагалось, что коэффициенты температуро- и теплопроводности стали остаются в процессе охлаждения постоянными, В действительности же их значения с изменением температуры меняются. Однако решение задачи с учетом функциональной зависимости этих величин от температуры представляется крайне сложным. Поэтому в дальнейшие расчеты вводится усредненный коэффициент температуропроводности, равный для стали $12 \mathrm{X} 1 \mathrm{M \Phi} a=$ $=0 ; 93 \cdot 10^{-5} \mathrm{~m}^{2} /$ сек. Это значение с удовлетворительной точностью может быть принято постоянным в пределах от 400 до $550{ }^{\circ} \mathrm{C}$ в начальном состоянии образца и до $150^{\circ} \mathrm{C}$ - в охлажденном. Коэффициент же теплопроводности $\lambda$ в любом случае согласуется с величиной $\alpha r_{0}^{2}$, и температура, при которой он выбирается, не имеет существенного значения. В настоящей работе для стали $12 \mathrm{X} 1 \mathrm{M}$ п прнято $\lambda=41,3 \mathrm{BT} /($ M.K) .

c. На.рис. 3: показаны определенные из опытов значения $\Delta t(0, \tau)$. - $\left(\alpha r_{0}^{2} \delta t\right)^{-1}$ в зависимости от времени. Кривые рассчитаны по выражению (5) при $\tau_{*}=0,05$ сек, т. е. как $\operatorname{arctg} \sqrt{\tau / 0,05}\left(2 \lambda \sqrt{\pi a 0,05)^{-1}}\right.$. Видно хорошее согласие опытных значений $\Delta t(0, \tau)$ с зависимостью $\operatorname{arctg} \sqrt{\tau / \tau *}$. 2...-Как уже отмечалось выше, недостатком известных формул температурного поля при резком охлаждении металла трубы является невозможность выражения их через элементарные функции. Однако в рассмӓриваемом случае, зная частное решение (5) и учитывая, что расғределение температуры по толщине стенки трубы отвечает экспо- 

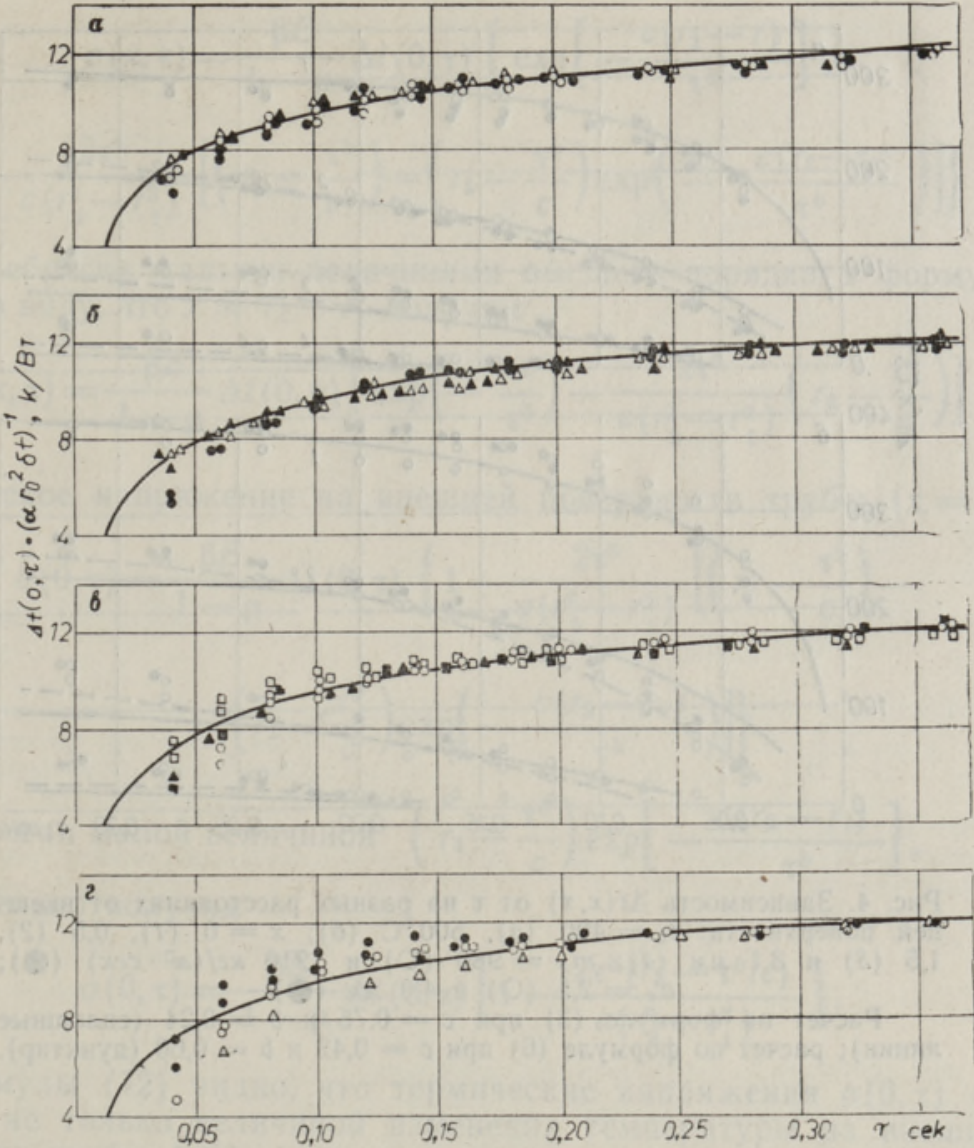

Рис. 3. Зависимость $\Delta t(0, \tau) \cdot\left(\alpha r_{0}^{2} \delta t\right)^{-1}$ от $\tau: t_{0}=400(a), 450$ (б),

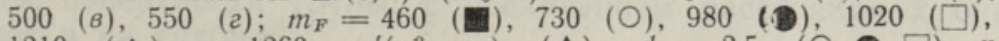

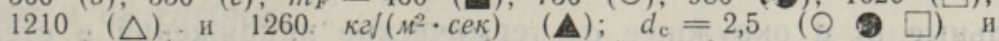
$4,0 \mathrm{MM}(\triangle \mathbf{\Delta})$.

ненциальному закону (2), можно представить температурное поле в полуэмпирическом виде

$$
\Delta t(x, \tau)=\Delta t(0, \tau) \cdot \exp \left(-c x / \tau^{b}\right),
$$

где постоянные $c$ и $b$ определяются в результате аппроксимации выражения (3) или опытных данных.

Расчеты на ЭВМ показали, что выражение (6) с большой точностью описывает закономерность изменения температуры по толщине стенки (3) при $c=0,75$ и $b=0,24$. Расчеты температурного поля-в стенке трубы по. выраженйо (3) изображены в виде сплошных линий на рис. 4 (такие же результаты получаются при использовании выражения (6) и тех же коэффициентах $c$ и $b$ ). Как видно, расчетные и экспериментальные данные удовлетворительно совпадают.

В результате аппроксимации опытных данных выражением (6) (на ЭВМ) получено $c=0,42$ и $b=0,60$. Рассчитанное по этим значениям температурное поле в стенке трубы показано на рис. 4 пунктирными линиями. 


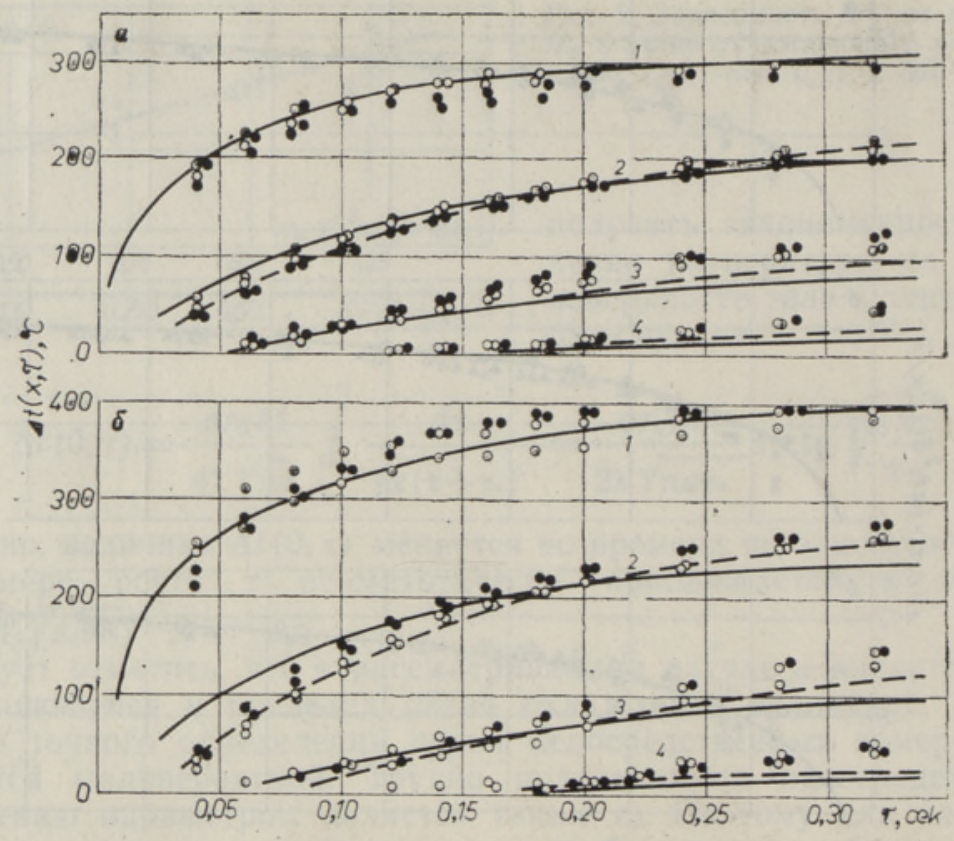

Рис. 4. Зависимость $\Delta t(x, \tau)$ от $\tau$ на разных расстояниях от внешней поверхности: $t_{0}=400(a), 500^{\circ} \mathrm{C}$ (б); $x=0$ (1), 0,5 (2), 1,5 (3) н 3,1 мм (4); $m_{F}=980$ (О) и $1210 \mathrm{\kappa z} /\left(\omega^{2} \cdot\right.$ сек) $d_{\mathrm{c}}=2,5$ (О) и 4,0 м. (อ).

Расчет по формуле (3) при $c=0,75$ и $b=0,24$ (сплошные линии); расчет по формуле (6) при $c=0,42$ и $b=0,60$ (пунктир).

\section{Термические напряжения в стенке трубы при ее резком охлаждении}

Аксиальные $\sigma_{z}$ и окружные $\sigma_{\varphi}$ термические напряжения в стенке неравномерно нагретой трубы могут быть определены по формулам

$$
\sigma_{z}=-\frac{\beta E}{1-\mu}\left[\Delta t(r, \tau)-\frac{2}{r_{2}^{2}-r^{2}} \int_{1 r_{1}}^{r_{2}} r \Delta t(r, \tau) d r\right]
$$

И

$$
\sigma_{\varphi}=\frac{\beta E}{(1-\mu) r^{2}}\left[\frac{r^{2}+r_{1}^{2}}{r_{2}^{2}-r_{1}^{2}} \int_{r_{1}}^{r_{2}} r \Delta t(r, \tau) d r+\int_{r_{1}}^{r_{2}} r \Delta t(r, \tau) d r-r^{2} \Delta t(r, \tau)\right]
$$

соответственно, где $\beta$ - коэффициент линейного расширения металла, $E$ - модуль упругости металла, $\mu$ - коэффициент Пуассона, $r-$ текущее значение радиуса трубы.

Радиальные термические напряжения $\sigma_{r}$ ввиду их малости по сравнению с $\sigma_{z}$ и $\sigma_{\varphi}$ здесь не рассматриваются. На внешней поверхности трубы $\sigma_{z}=\sigma_{\varphi}$. А поскольку и при $r<r_{2}$ окружные напряжения практически не отличаются от аксиальных, то с большой степенью точности можно ограничиться только формулой (7). Тогда с учетом формулы (6) имеем (в дальнейшем индекс $z$ опущен) 


$$
\begin{gathered}
\sigma(x, \tau)=\frac{\beta E}{1-\mu} \Delta t(0, \tau)\left\{\exp \left[-\frac{c\left(r_{2}-r\right)}{\tau^{b}}\right]-\right. \\
\left.-\frac{2 \tau^{b}}{c\left(r_{2}^{2}-r_{1}^{2}\right)}\left[\left(r_{2}-\frac{\tau^{b}}{c}\right)-\left(r_{1}-\frac{\tau^{b}}{c}\right) \exp \left(-\frac{c\left(r_{2}-r_{1}\right)}{\tau^{b}}\right)\right]\right\} .
\end{gathered}
$$

Пренебрегая малыми величинами высшего порядка в формуле (9) и имея в виду, что $x=r_{2}-r$, получим

$$
\sigma(x, \tau)=\frac{\beta E}{1-\mu} \Delta t(0, \tau)\left[\exp \left(-\frac{c x}{\tau^{b}}\right)-\frac{2 \tau^{b}}{c\left(r_{2}^{2}-r_{1}^{2}\right)}\left(r_{2}-\frac{\tau^{b}}{c}\right)\right] .
$$

Термическое напряжение на внешней поверхности трубы $(x=0)$

$$
\begin{aligned}
\sigma(0, \tau)= & \frac{\beta E}{1-\mu} \Delta t(0, \tau)\left\{1-\frac{2 \tau^{b}}{c\left(r_{2}^{2}-r_{1}^{2}\right)}\left[\left(r_{2}-\frac{\tau^{b}}{c}\right)-\right.\right. \\
& \left.\left.-\left(r_{1}-\frac{\tau^{b}}{c}\right) \exp \left(-\frac{c\left(r_{2}-r_{1}\right)}{\tau^{b}}\right)\right]\right\} .
\end{aligned}
$$

Пренебрегая малой величиной $\left(r_{1}-\frac{\tau^{b}}{c}\right) \exp \left[-\frac{c\left(r_{2}-r_{1}\right)}{\tau^{b}}\right]$, окончательно получим

$$
\sigma(0, \tau)=\frac{\beta E}{1-\mu} \Delta t(0, \tau)\left[1-\frac{2 \tau^{b}\left(r_{2}-\tau^{b} / c\right)}{c\left(r_{2}^{2}-r_{1}^{2}\right)}\right] .
$$

Из формулы (12) видно, что термические напряжения $\sigma(0, \tau)$ определяются не только величиной изменения температуры на внешней поверхности трубы $\Delta t(0, \tau)$, но и величинами $\tau, r_{1}$ и $r_{2}$. Результаты расчета показывают, что эти напряжения являются максимальными на внешней поверхности трубы, с увеличением расстояния $x$ они резко убывают и на глубине

$$
x_{0}=-\ln \left[2 \tau^{b}\left(r-\frac{\tau^{b}}{c}\right) / c\left(r_{2}^{2}-r_{1}^{2}\right)\right]
$$

становятся равными нулю (для стали $12 \mathrm{X} 1 \mathrm{MФ}-$ при $x=1,7$ мм и $\tau=0,2$ сек).

В качестве примера на рис. 5 приведены результаты расчета термических напряжений для стали $12 \mathrm{X} 1 \mathrm{MФ.} \mathrm{На} \mathrm{этом} \mathrm{же} \mathrm{рисунке} \mathrm{нане-}$ сена вероятная область изменения двукратного значения предела текучести стали $12 X 1 M \Phi$, превышение которого может вызвать образование трещин термической усталости [ $\left.{ }^{4}\right]$. Отметим, что этот предел, по данным различных источников, колеблется в широких пределах.

На рис. 6 изображена зависимость термических напряжений на внешней поверхности трубы от времени при различных первоначальных температурах металла. Как видно, при $\tau \leqslant 0,2$ сек результаты расчетов при коэффициентах $c=0,75, b=0,24$ и $c=0,42, b=0,60$ практически совпадают. Их некоторое расхождение начинается с $\tau>0,2$ сек, что можно объяснить влиянием конечных размеров толщины стенки, которые не учтены в исходной расчетной схеме. Это расхождение невелико и при $\tau=0,25$ сек составляет примерно $2 \%$ и при $\tau=0,3$ секоколо $5 \%$. 


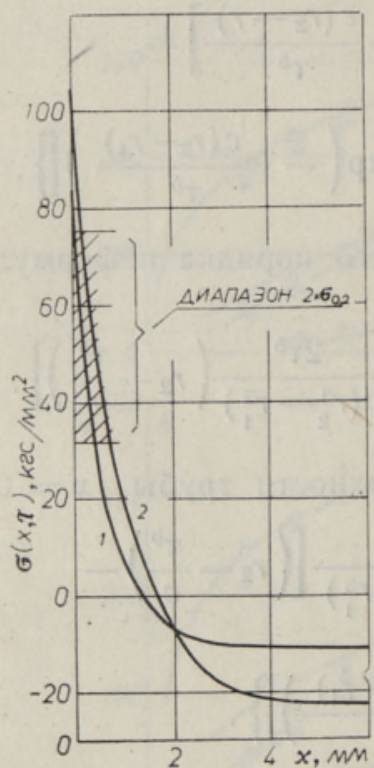

Рис. 5. Изменение аксиального термического напряжения по глубине стенки трубы при $t_{0}=500^{\circ} \mathrm{C}$ : $\tau=0,1$ (1) и 0,2 сек (2).

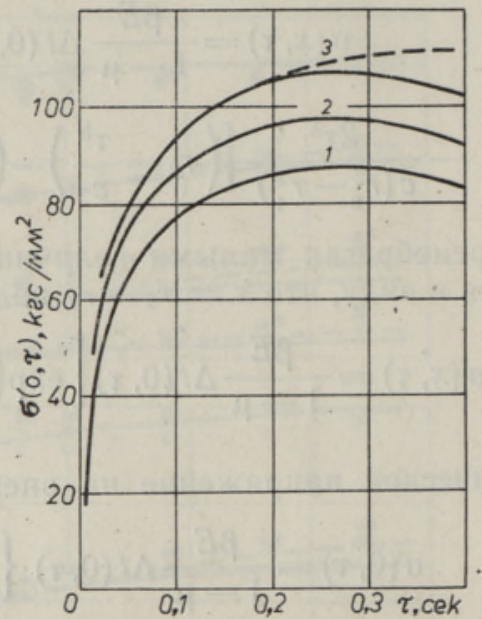

Рис. 6. Зависимость термических напряжений на внешней поверхности трубы от времени: $\begin{array}{llll}t_{0}=400 & (1), & 450 & (2) \\ 500^{\circ} \mathrm{C} & (3) .\end{array}$

Расчет по формуле (12) при $c=0,42$ и $b=0,60$ (сплошные линии); то же при $c=0,75$ и $b=0,24$ (пунктир).

Из рис. 6 выясняется, что на внешней поверхности трубы термические напряжения очень быстро растут во времени и, например при указанных выше условиях, уже менее чем через 0,025 сек после начала охлаждения достигают двукратного предела текучести материала $2 \sigma_{\theta, 2}$. По достижении максимума они практически не меняются с ростом времени, хотя температура на внешней поверхности продолжает снижаться. Время, при котором термические напряжения достигают максимальных значений, можно определить из условия

$$
\begin{gathered}
\frac{d \sigma(0, \tau)}{d \tau}=\frac{\sqrt{\tau}}{2 \sqrt{\tau}\left(\tau+\tau_{*}\right)}\left[1-\frac{2 \tau^{b}\left(r_{2}-\frac{\tau^{b}}{c}\right)}{c\left(r_{2}^{2}-r_{1}^{2}\right)}\right]- \\
-\operatorname{arctg} \sqrt{\frac{\tau}{\tau \cdot}}\left[\frac{2 b \tau^{b-1}}{c\left(r_{2}^{2}-r_{1}^{2}\right)}\left(r_{2}-\frac{2 \tau^{b}}{c}\right)\right]=0 .
\end{gathered}
$$

Расчеты показывают, что для трубы из стали 12 Х1МФ это время составляет 0,25 сек.

\section{Критерии надежной работы металла в условиях резкого охлаждения}

Оценивая надежность и долговечность работы металла в рассматриваемых выше условиях, следует различать три случая:

1. Термоциклические деформации в упругой области. 


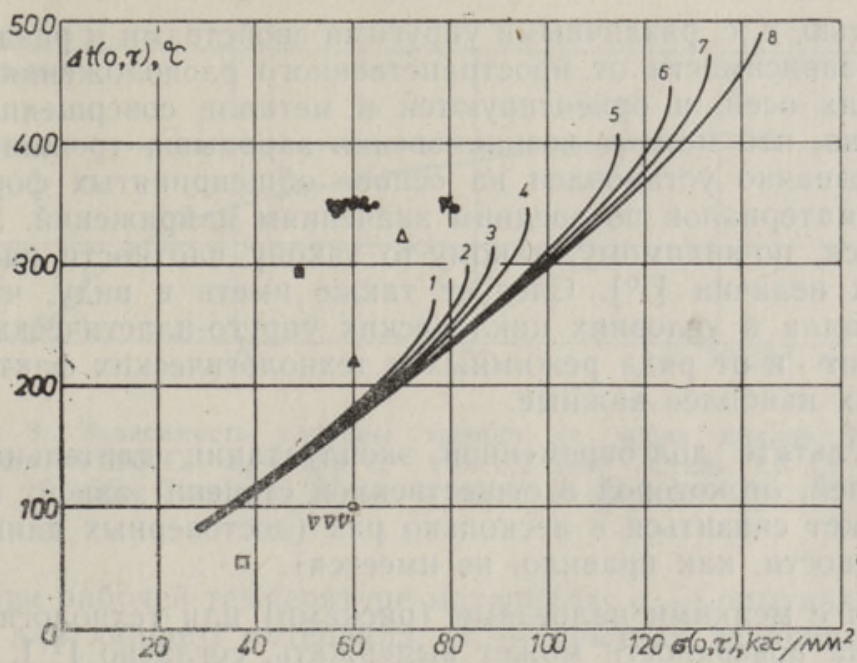

Рис. 7. Зависимость $\sigma(0, \tau)$ от $\Delta t(0, \tau): \delta t=400(1-4)$ и $600^{\circ} \mathrm{C}(5-8) ; m_{F}=375(1,5), 700(2,6), 1000(3,7)$ и $1400 \mathrm{\kappa a/}(\mathbf{f}$ - - сек) $(4,8)$. По данным авторов (8) $)$, по

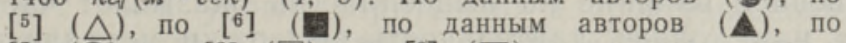
$\left[{ }^{7}\right](0)$, по $\left[{ }^{8}\right](\square)$, по $\left.{ }^{[9}\right](\nabla)$, по данным авторов

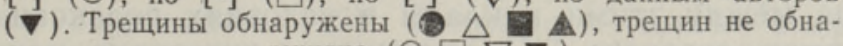
ружено $($ о $\square \nabla \nabla)$.

2. Термоциклические деформации в упруго-пластической области без образования трещин термической усталости.

3. Термоциклирование в условиях развивающихся трещин.

При циклических знакопеременных термических напряжениях работа металла без пластических деформаций, а следовательно, и без малоцикловой усталости, обеспечивается при выполнении условия $\left[{ }^{4}\right]$ :

$$
\sigma(0, \tau) \leqslant 2 \sigma_{0,2}
$$

где $\sigma(0, \tau)$ может быть определено по формуле (12).

Рис. 7 иллюстрирует зависимость $\sigma(0, \tau)$ от $\Delta t(0, \tau)$ для труб диаметром 32 м. из стали $12 \mathrm{X} 1 \mathrm{M \Phi} \mathrm{при} \mathrm{разных} \mathrm{значениях} m_{F}$, $\delta t$ и длительности охлаждения 0,2 сек. Точки соответствуют условиям опытных исследований труб на термоциклическое растрескивание при их резком поверхностном охлаждении. Выявилось, что во всех случаях, когда $\sigma(0, \tau)>2 \sigma_{0,2}$, на поверхностях труб появляются трещины. Из 17 опытных труб только одна не имела трещин и именно у нее $\sigma_{0,2}$ превышало среднее значение $\sigma_{0,2}$ остальных труб примерно на $33 \%$.

Принимая, например, для стали $12 \mathrm{X} 1 \mathrm{M} Ф$ значение $\sigma_{0,2}$ при 450 $480^{\circ} \mathrm{C}$ равным $27 \kappa 2 c / \mu \mu^{2}$, получим, что безопасной с точки зрения растрескивания металла $\Delta t(0, \tau) \leqslant 150-160^{\circ} \mathrm{C}$. Этой величине соответствует длительность охлаждения 0,02 сек.

Известно, что в некоторых случаях металл может выдержать некоторое количество циклов загружения в условиях незначительных пластических деформаций. Однако при этом его выносливость определяется очень сложными, как правило, не поддающимися точному учету факторами. Как показывают исследования, зародыш трещины возникает в кристаллах металла на той стадии деформации, когда касательные напряжения в них впервые достигают порогового уровня, превышающего прочность зерен. Поскольку зерна металла обладают ани- 
зотропностью, т. е. различными упругими свойствами и различной прочностью в зависимости от пространственного расположення кристаллографических осей, и ориентируются в металле совершенно случайно, то очевидно, что момент возникновения зародыша трещины не может быть однозначно установлен на основе общепринятых формул сопротивления материалов по средним значениям напряжений. Это явление подчиняется, по-видимому, какому-то закону плотности распределения случайных величин $\left[{ }^{10}\right]$. Следует также иметь в виду, что выносливость металла в условиях циклических упруго-пластических деформаций зависит и от ряда режимных и технологических факторов. Отметим из них наиболее важные.

1) В результате долговременной эксплуатации длительная пластичность сталей, от которой в существенной степени зависит их выносливость, может снизиться в несколько раз (достоверных данных об этой закономерности, как правило, не имеется).

2) Металл с мелкими надрезами (рисками) или технологическими дефектами на поверхности может выдержать, согласно [11], без образования трещин до 20 раз меньше циклов упруго-пластических деформаций, чем металл, имеющий гладкую поверхность.

3) Существенное влияние на выносливость оказывает частота циклов загружения или, точнее, длительность выдержки металла при высокой температуре между этими циклами в условиях релаксации напряжений. Например, согласно $\left[{ }^{12}\right]$, увеличение интервала между циклами от 15 до 85 ч приводит к уменьшению циклов, выдерживаемых образцом до разрушения, в 3,7 раза. В свете сказанного очевидно, что аналитическое определение их количества не может дать адекватных опытам результатов. Қак показывает анализ, разработанные к настоящему времени методы аналитических расчетов $\left[{ }^{13,14}\right]$ не могут гарантировать необходимую долговечность работы металла без возникновения трещин даже в случае принятых коэффициентов запаса по числу циклов до 20.

В то же время из практики известно, что даже в условиях постоянных по амплитуде циклических напряжений детали (лопатки турбин, оси железнодорожных вагонов и др.) могут работать после появления усталостных трещин в несколько раз дольше, чем до их появления. Поэтому представляется важным рассмотреть закономерность распространения возникшей на поверхности металла трещины в условиях термоцикличности при $\sigma(0, \tau)>2 \sigma_{0,2}$. Задача несколько упрощается в связи с тем, что эта закономерность определяется нормальными напряжениями возле вершины трещины, которые в условиях пластических деформаций близки к статистически усредненным напряжениям отдельных зерен металла, сравнительно просто определяемым обычными методами сопротивления материалов и теории упругости. Так как при кратковременном резком охлаждении поверхности термические напряжения очень резко убывают с увеличением расстояния от поверхности (рис. 5), то, очевидно, по мере углубления трещины скорость ее роста должна, быстро затухая, стремиться к нулю.

В основу зависимости, отражающей закономерность развития трещин термоциклической усталости, может быть принято выражение из $\left[{ }^{15}\right]$ :

$$
N=\left(1-a / a_{0}\right) / A\left(\sigma / \sigma_{b}^{t}\right)^{\mu},
$$

где $N$ - число циклов; $a_{0}$ - глубина исходной трещины (начало отсчета); $a$ - глубина трещины при количестве циклов $N ; \sigma_{b}{ }^{t}-$ предел 


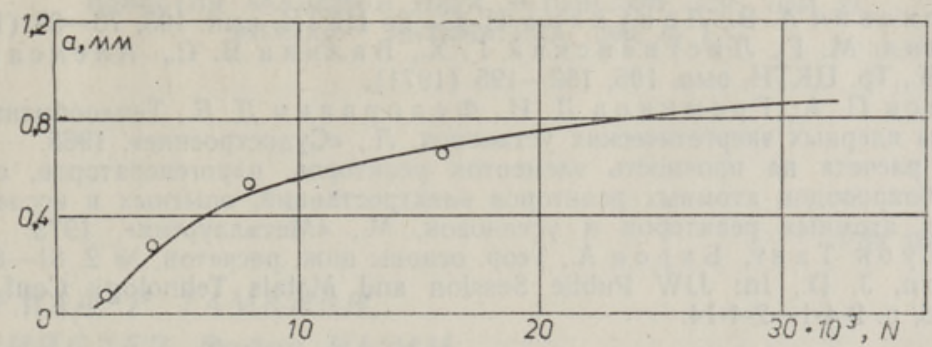

Рис. 8. Зависимость глубины трещин от числа циклов $N$ : $\Delta t(0, \tau)=290^{\circ} \mathrm{C}, \quad t_{0}=550^{\circ} \mathrm{C}, \quad \tau=0,2$ сек. Сталь $1,0 \mathrm{Cr}$ и 0,5 Мо. Сплошная линия - расчет по формуле (16).

прочности при рабочей температуре материала; $\mu$ - опытная величина, постоянная для данного материала; $A$ - опытная величина, постоянная для данного материала при его охлаждении с неизменяющейся частотой.

Наши исследования показывают, что опытная зависимость $a=$ $=F(N)$ может быть удовлетворительно аппроксимирована выражением (16), если вместо б подставить ее функциональную зависимость от $x$ согласно выражению (10). Результаты такой аппроксимации опытных значений $a$, полученных в $\left[{ }^{16}\right]$, показаны на рис. 8 . Видно, что в начальный период трещина термической усталости действительно растет относителіьно быстро, но по мере углубления скорость ее распространения стремится к нулю, т. е. процесс является в рассматриваемых условиях самотормозящимся.

Прогнозируя в соответствии со сказанным закономерность распространения трещин и учитывая это при проектировании, можно обеспечить необходимую надежность и длительную работоспособность металла в условиях термоцикличности при упруго-пластических дефор-

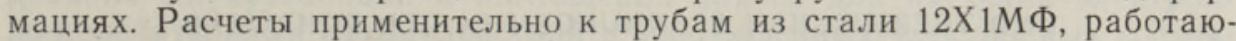
щим в указанном выше режиме, показывают, например, что при глубине трещин $a \leqslant 0,5$ мм и количестве термоциклов за срок службы $N=15000$ допустимая величина $\Delta t(0, \tau)$ составит примерно $285^{\circ} \mathrm{C}$ (вместо $150-160^{\circ} \mathrm{C}$ при соблюдении условия (15)). Это обстоятельство является весьма важным, например, с точки зрения эффективности водяной обмывки поверхностей нагрева парогенераторов электростанций, определяемой величиной создаваемого температурного перепада в металле, т. е. допустимым значением $\Delta t$ на поверхности металла.

\section{ЛИТЕРА Т У А}

1. Л ы ко в В. А., Теория теплопроводности, М., «Высш. школа», 1967.

2. Boley, B. A., Weiner, J. H., Theory of thermal stresses, John Wiley and Sons, Inc., New York-London, 1968.

3. Р ы к а и н Н. Н., Расчеты тепловых процессов при сварке, М., Машгиз, 1951.

4. Отс А. А., Ансон П. И., Т аллермо Х. И., Тр. Таллинск. политехн. ин-та, XVIII, № 458, 47-57 (1978)

5. Туляков Г. А., Плех анов В. А., Теплоэнергетика, № 4, 45-49 (1969).

6. Эл ле р и А. Р., Д жон сон П. Р., Н ьютон Д. О., Энерг. машины и установки, № 4, 62-69 (1974).

7. Кендысь П. Н., Кельм ан Л. Я., Энергомашиностроение, № 2, 1 -3 (1971)

8. Nels o n, J. E., In: Proc. Amer. Power Conf., 37, Chicago, 1975, p. 410-420.

9. Nelson, J. E., Desiderio, A. J., B uckley, W. R., In: Proc. Amer. Power Conf., 33, Chicago, 1971, p. 584-593.

10. Их а а, Цур уи, Й а раши, Теор. основы инж. расчетов, № 3, $21-23$ (1977). 
11. Ст ан юкович А. В., Л а пухин а Н. С., Тр ЦКТИ, вып. 105, 73-82 (1971).

12. Т ау бина М. Г., Листвинский Г. Х., Балина В. С., Александрова Л. В., Тр. ЦКТИ, вып. 105, 189-195 (1971).

13. Анд реев П. А., Грем инов Д. И., Федорович Д. Е., Теплообменные аппараты ядерных энергетических установок, Л., «Судостроение», 1965.

14. Нормы расчета на прочность элементов реакторов, парогенераторов, сосудов и трубопроводов атомных реакторов электростанций, опытных и исследовательских атомных реакторов и установок, М., «Металлургия», 1973.

15. Буи - Куок Т анг, Би рон А., Теор. основы ннж, расчетов, № 2, $81-87$ (1975).

16. Newton, J. D., In: JJW Public Session and Metals Tehnalogy Conf., Sydney, 1976, p. 2-4-1-2-4-14.

Таллинский политехнический институт

Поступила в редакцию 2/VII 1979

A. OTS, P. ANSON, U. SOODLA, H. TALLERMO

\section{KIIRESTI JAHUTATAVA TORU TEMPERATUURIVÃLJA JA TERMILISTE PINGETE LIGIKAUDSE ARVUTAMISE MEETOD}

Esitatud meetod põhineb soojusvoogude määramisel toru lokaalse soojendamise (jahutamise) korral. Temperatuurivälja integraalvōrrandi osalahendist on saadud praktilisteks arvutusteks sobiv poolempiiriline vōrrand. On võrreldud veega jahutatud terastoru $(12 X 1 M \Phi)$ seinas katseliselt määratud temperatuurivälju analüütiliste arvutuste tulemustega. Temperatuurivälja valemite alusel on esitatud seosed termiliste pingete määramiseks ja analüüsitud kriteeriume, mis vōimaldavad hinnata kuumutatud ja veejoaga jahutatavate torude töökindlust termiliste väsimuspragude tekke seisukohast.

\section{A. OTS, P. ANSON, U. SOODLA, H. TALLERMO}

\section{NÄHERUNGSMETHODE ZUR BERECHNUNG VON TEMPERATURFELDERN UND THERMISCHEN SPANNUNGEN IN DER ROHRWAND BEI PLOTZLICHER ABKOHLUNG}

Im Artikel wird die Bestimmung von Temperaturfeldern in erhitzten Stahlzylindern und Rohren bei kurzzeitiger Abkühlung ihrer Oberfläche durch Flüssigkeiten betrachtet. Auf der Grundlage der Berechnungsmethode von Wärmeströmen unter den Bedingungen örtlicher Aufheizung (Abkühlung) wurde eine Integralformel zur Berechnung der Wärmefelder aufgestellt, mit deren Hilfe praktische Teillösungen (halbempirisch) möglich sind. Aufgeführt werden experimentelle Ergebnisse bei kurzfristiger Abkühlung von Kesselstahl 12X1M $\Phi$ bei unterschiedlichen Anfangstemperaturen im Vergleich zu der analytischen Betrachtung.

Im Ergebnis werden die Kriterien der Zuverlässigkeit der Metalle aufgewärmter Rohre bei Abkühlung ihrer Oberflächen durch Wasser aufgezeigt. Das Hauptkriterium der Zuverlässigkeit bei der Arbeitsphase der Metalle ohne Rißbildung ist die Bedingung, bei der die thermischen Spannungen etwa $2 \mathrm{mal}$ niedriger als die Fließgrenze des Metalls sind.

Außerdem werden die Gesetzmäßigkeiten der Rißbildung bei thermischer Ermüdung der Metalle betrachtet. Als Schlußfolgerung ergibt sich, daß dieser Prozeß «selbstbremsend» abläuft und mit Hilfe der vorgeschlagenen Berechnungsmethode, bei möglicher Rißbildung bis in vorgegebene Metalltiefe, eine Prognose der langwierigen Arbeitsphase dieser Metalle möglich ist. 